\title{
Associations between longevity of parents and glucose regulation in their offspring: the KORA S4/F4 Study
}

\author{
B. Kowall • A. Peters • B. Thorand • W. Rathmann • \\ C. Meisinger
}

Received: 25 July 2012 / Accepted: 18 September 2012 / Published online: 18 October 2012

(C) Springer-Verlag Berlin Heidelberg 2012

\begin{abstract}
Aims Type 2 diabetes was less prevalent in studies of the offspring of centenarians and a separate study of nonagenarian siblings. We examined whether this reduction would also be found when less extreme criteria of parental longevity (a lifespan of at least 80 years) were applied. Moreover, we looked for an association between parental longevity and incidence of dysglycaemia, which has not yet been reported for a population-based study group.

Methods Baseline and 7-year follow-up data on 55-74year-old participants in the population-based German Cooperative Health Research in the Region of Augsburg (KORA) S4/F4 cohort study were used for the analyses. Participants whose parents had died from traumatic causes were excluded. Diabetes was assessed by validated physician diagnosis or OGTTs. Using logistic regression models, adjusted OR and $95 \%$ CIs were calculated for the associations between parental longevity and the prevalence or incidence of dysglycaemia, which was defined as including either type 2 diabetes or prediabetes (defined in this study as comprising impaired glucose tolerance [IGT] and impaired fasting glucose [IFG]).

Results In age- and sex-adjusted models, the prevalence of type 2 diabetes was lower in individuals with one (OR 0.63 ,
\end{abstract}

W. Rathman and C. Meisinger shared senior authorship of this article.

B. Kowall $(\bowtie) \cdot$ W. Rathmann

Institute of Biometrics and Epidemiology,

German Diabetes Center, Leibniz Center for Diabetes

Research at Heinrich Heine University Düsseldorf,

Auf'm Hennekamp 65,

40225 Düsseldorf, Germany

e-mail: bernd.kowall@ddz.uni-duesseldorf.de

\footnotetext{
A. Peters $\cdot$ B. Thorand $\cdot$ C. Meisinger

Helmholtz Zentrum München,

German Research Center for Environmental Health, Institute of Epidemiology II,

Neuherberg, Germany
}

95\% CI $0.43,0.93$ ) or two (OR $0.46,95 \%$ CI $0.25,0.85$ ) long-lived parents. Among participants with normal glucose tolerance at baseline, the odds of incident dysglycaemia were lower in those with one (OR $0.65,95 \%$ CI 0.40 , 1.03 ) or two long-lived parents (OR $0.46,95 \%$ CI 0.22 , 0.96) after adjustment for age and sex.

Conclusions/interpretation This study showed that longevity of the parents, defined by a lifespan of at least 80 years, was associated with a lower prevalence and incidence of dysglycaemia in their offspring in an older German population.

Keywords Diabetes mellitus · Longevity · Offspring · Parents

\begin{tabular}{|c|c|}
\hline \multicolumn{2}{|c|}{ Abbreviations } \\
\hline IFG & Impaired fasting glucose \\
\hline IGT & Impaired glucose tolerance \\
\hline KORA & $\begin{array}{l}\text { Cooperative Health Research in the Region } \\
\text { of Augsburg }\end{array}$ \\
\hline NGT & Normal glucose tolerance \\
\hline SES & Socioeconomic status \\
\hline
\end{tabular}

\section{Introduction}

It has been well established that type 2 diabetes is associated with premature death [1], and that long-lived individuals are likely to have a genetic and environmental protection against type 2 diabetes [2]. Transmission of this protection to the descendants of long-lived families might lead to a lower risk of diabetes in the offspring.

A reduced prevalence of type 2 diabetes has been observed in three cross-sectional studies in the offspring of extremely long-lived families. In the Leiden Longevity Study, the offspring of nonagenarian siblings were compared with those of their partners, the offspring displaying 
a lower prevalence of diabetes, lower levels of fasting glucose, and better glucose tolerance [3, 4]. In the offspring of Ashkenazi Jewish centenarians, the prevalence of diabetes was reduced by about $50 \%$ compared with the offspring's spouses and other control groups [5]. Terry et al compared the offspring of centenarians with the offspring of parents who were born in the same year as the centenarians but had died at an average age $[6,7]$. In the offspring of centenarians, the prevalence of diabetes was reduced by $59 \%$ [6], and the onset of diabetes was delayed by 8.5 years [7]. In a population-based Danish survey with participants aged over 70, a significant association between the mean age at death of the participants' parents and the prevalence of diabetes of the participants was found [8]. In two other population-based Danish surveys with middle-aged or nonagenarian individuals, this association was not statistically significant [8].

So far, only one study has been published on the association between parental longevity and incident diabetes [9]. In the Diabetes Prevention Program, including only participants with a high risk of type 2 diabetes, both paternal and maternal longevity, defined by a lifespan of at least 80 years, were associated with a reduction of diabetes risk of roughly $30 \%$.

The objectives of the current study were: (1) to investigate whether a cross-sectional association between parental longevity and dysglycaemia would also be found when less extreme criteria for longevity were used; and (2) to assess whether parental longevity was associated with a lower risk of dysglycaemia in a population-based sample.

\section{Methods}

Study population for cross-sectional analyses The populationbased Cooperative Health Research in the Region of Augsburg (KORA) S4 Survey was carried out in Southern Germany in the same region and using the same methods as the previous WHO Monitoring Trends and Determinants in Cardiovascular Disease Augsburg (MONICA) project. The study design, sampling method and data collection have been described in detail elsewhere [10]. The study participants gave informed consent, and the study was approved by the Ethics Committee of the Bavarian Physician Chamber.

In the population-based KORA S4 survey, carried out in 1999-2001, 2,656 participants aged 55-74 years were invited for baseline investigations. Overall, 1,653 (62\%) participated. Out of these, 187 individuals could not be included in the present analyses, mainly because they were not able to attend the study centre during the morning hours. Of the remaining 1,466, participants were included in the analyses related to parental longevity if the following conditions were fulfilled:

(1) Their father (and/or mother) had not died from traumatic causes of death (war, accident, murder, hunger);

(2) Their father (and/or mother) had already died and their age at death was known, or if their father (and/or mother) were still alive, the study participant was at least 65 years old. In the latter case, we assumed that father (and/or mother) was at least 80 years old, and thus long-lived according to our definition of longevity.

For analyses related to paternal longevity and prevalent diabetes, 1,108 participants met the inclusion criteria. For analyses related to maternal longevity and parental longevity 1,218 and 969 individuals, respectively, were included.

Study population for longitudinal analyses Of the 1,653 participants in the KORA S4 study, 1,353 participants had had an OGTT at baseline. This cohort was reinvestigated between 2006 and 2008 (the KORA F4 survey). Among the 1,202 participants without known or newly diagnosed diabetes at baseline and who still lived within the study region, 887 (74\%) participated in the follow-up. For 592 individuals, data on parental longevity were available; of these, 429 (72\%) had normal glucose tolerance (NGT) at baseline and $163(28 \%)$ had prediabetes (defined in this study as comprising impaired glucose tolerance [IGT] and impaired fasting glucose $[\mathrm{IFG}])$.

Parental age at death and definition of longevity Participants were asked whether their parents were still alive, and if not, how old their parents had been when they died and what the cause of death had been (the categories being diabetes, myocardial infarction, stroke, cancer, killed at war, accident, other causes, and don't know). Those who marked the category 'other causes' were asked to specify the cause of death of their parents. Parents were defined as long-lived (1) if their age at death had been at least 80 years, or (2) if they were still alive and the study participants were at least 65 years old.

Ascertainment of diabetes and prediabetes In the KORA S4 and F4 surveys, self-reports of diabetes were validated by contacting the general practitioners who were treating the participants. At both baseline and follow-up, OGTTs were performed during the morning (7:00-11:00 hours) for those who did not have diabetes. Participants were asked to fast for at least $10 \mathrm{~h}$ overnight, to avoid heavy physical activity on the day before examination and to refrain from smoking before and during the test. Exclusion criteria for the OGTT were acute illnesses (infection, fever, acute gastrointestinal diseases). Fasting venous blood glucose was sampled, and $75 \mathrm{~g}$ of anhydrous glucose was given (Dextro OGT, Roche Diagnostics, Mannheim, Germany). 
Prevalent and incident diabetes were defined based on: (1) validated physician diagnoses; or (2) newly diagnosed diabetes $(\geq 7.0 \mathrm{mmol} / 1$ fasting plasma glucose or $\geq 11.1 \mathrm{mmol} / 12$-h plasma glucose). Prediabetes comprised impaired glucose tolerance (IGT) and impaired fasting glucose (IFG). IGT was defined as 2-h plasma glucose $\geq 7.8$ but $<11.1 \mathrm{mmol} / \mathrm{l}$, and a fasting plasma glucose $<7.0 \mathrm{mmol} / \mathrm{l}$. IFG was defined as fasting plasma glucose $\geq 6.1$ but $<7.0 \mathrm{mmol} / 1$, and 2 -h plasma glucose $<7.8 \mathrm{mmol} / \mathrm{l}[11]$.

Physical examinations and interviews Height, weight, waist circumference and systolic and diastolic blood pressure were measured based on standard protocols as described elsewhere [10]. Medical interviewers gathered information on physical activity, alcohol consumption, smoking and parental history of diabetes.

Laboratory measurements Blood was collected without stasis. All blood variables except for 2 -h plasma glucose were based on fasting blood samples. After blood withdrawal, the blood samples were centrifuged and kept $\operatorname{cool}\left(4^{\circ} \mathrm{C}\right)$ until the plasma glucose was analysed in the central laboratory, which took place within a maximum of $6 \mathrm{~h}$ after withdrawal. Plasma glucose, LDL-cholesterol and HDL-cholesterol were determined as described elsewhere [10].

Statistical analyses Baseline characteristics were compared between participants with no, one and two long-lived parents. Global F-tests were used for comparison of metric variables, and logistic regressions were used for comparison of dichotomous variables. All comparisons were adjusted for age and sex.

Binomial logistic regression models were applied to calculate ORs with $95 \%$ CIs for the associations between longevity (number of long-lived parents, paternal or maternal longevity), and prevalent and incident dysglycaemia, respectively. Dysglycaemia was defined as either prediabetes or diabetes. Multinomial logistic regression analyses were fitted to calculate the associations between the number of long-lived parents as the independent variable, and glucose regulation (NGT [as the reference], prediabetes and diabetes) at baseline and at follow-up, respectively, as the dependent variable. For each type of regression analysis, three different models were fitted: a model adjusted for sex and age at baseline (model 1), a model additionally adjusted for BMI, socioeconomic status (SES), smoking, alcohol consumption, and physical activity (model 2), and a model additionally adjusted for parental diabetes with a diagnosis before the age of 60 years (model 3).

The covariables were categorised as follows: smoking: current smokers, former smokers or never smokers; high alcohol intake: $>40 \mathrm{~g} /$ day for men, $>20 \mathrm{~g} /$ day for women; physical activity: participants with less than $1 \mathrm{~h}$ of physical activity per week during their leisure time in summer or winter were classified as inactive. SES was assessed using a score developed for Germany by Helmert et al, as previously described [12].

As an additional analysis, we fitted logistic regression models to assess the association between the mean parental age at death (per 10 years) and prevalent or incident dysglycaemia, respectively.

\section{Results}

Participants with one or two long-lived parents were older, were less often current smokers and had higher HDLcholesterol levels than those without long-lived parents (Table 1). No significant differences were seen with respect to anthropometric measures, blood pressure, SES, physical activity and alcohol consumption. With an increasing number of long-lived parents, non-significant trends were seen towards lower $\mathrm{HbA}_{1 \mathrm{c}}$ values and towards a lower proportion of parents with an onset of diabetes before the age of 60 years.

In both participants with a long-lived father and those with a long-lived mother, the prevalence of dysglycaemia was lower than in those who did not have a long-lived father or mother, respectively, after adjustment for age and sex (OR $0.75,95 \%$ CI $0.57,0.98$ for paternal longevity vs no paternal longevity; OR $0.76,95 \%$ CI $0.60,0.96$ for maternal longevity vs no maternal longevity). After further adjustment for BMI, SES, lifestyle factors and early onset of diabetes in the parents, these associations were slightly attenuated and significant only at a borderline level.

The prevalence of type 2 diabetes ranged from $18.8 \%$ for participants without long-lived parents to $14.0 \%$ (with one long-lived parent) and $11.2 \%$ (with two long-lived parents) (Table 1). In multinomial logistic regression analyses, we observed a dose-effect relationship with significantly lower odds of having type 2 diabetes for individuals with one longlived parent (OR $0.63,95 \%$ CI $0.43,0.93$ ), as well as for those with two long-lived parents (OR $0.46,95 \%$ CI $0.25,0.85$ ) (Table 2). These associations were slightly attenuated after additional adjustment for potential mediator variables. Prediabetes was less prevalent in participants with long-lived parents, but this difference was not statistically significant in age- and sex-adjusted models (Table 2). The OR for the association between mean parental age at death (per 10-year increase) and prevalent dysglycaemia was borderline significant $(0.87$, 95\% CI 0.76, 1.006 after adjustment for age and sex).

Participants with NGT at baseline had a significantly reduced risk of incident dysglycaemia when they had two long-lived parents (OR 0.46, 95\% CI 0.22, 0.96, with adjustment for age and sex) (Table 3). The reduction in risk 
Table 1 Baseline characteristics of KORA S4/F4 participants according to the number of long-lived parents

\begin{tabular}{|c|c|c|c|c|}
\hline Variable & $\begin{array}{l}\text { No parent long-lived } \\
(n=377)\end{array}$ & $\begin{array}{l}\text { One parent long-lived } \\
(n=458)\end{array}$ & $\begin{array}{l}\text { Both parents long-lived } \\
(n=134)\end{array}$ & $p$ value $^{\mathrm{a}}$ \\
\hline Age & $64.4 \pm 5.5$ & $65.4 \pm 5.3$ & $66.1 \pm 4.9$ & $<0.01$ \\
\hline Sex (male), $n(\%)$ & $208(55.2)$ & $229(50.0)$ & $74(55.2)$ & 0.25 \\
\hline BMI, $\mathrm{kg} / \mathrm{m}^{2}$ & $29.0 \pm 4.5$ & $28.6 \pm 4.1$ & $28.6 \pm 4.4$ & 0.26 \\
\hline Waist circumference, $\mathrm{cm}$ & $97.6 \pm 12.0$ & $95.9 \pm 11.4$ & $96.6 \pm 12.0$ & 0.11 \\
\hline Systolic blood pressure, $\mathrm{mmHg}$ & $136.8 \pm 20.6$ & $137.5 \pm 20.1$ & $137.0 \pm 19.6$ & 0.75 \\
\hline Diastolic blood pressure, $\mathrm{mmHg}$ & $80.2 \pm 10.7$ & $80.3 \pm 10.0$ & $80.0 \pm 10.5$ & 0.75 \\
\hline Hypertension, $n(\%)^{\mathrm{b}}$ & $211(56.0)$ & $282(61.6)$ & $77(57.5)$ & 0.29 \\
\hline LDL-cholesterol, mmol/1 & $3.95 \pm 1.00$ & $3.96 \pm 1.04$ & $4.02 \pm 0.89$ & 0.78 \\
\hline HDL-cholesterol, mmol/1 & $1.45 \pm 0.42$ & $1.52 \pm 0.42$ & $1.57 \pm 0.41$ & $<0.01$ \\
\hline $\mathrm{SES}^{\mathrm{c}}$ & $12.6 \pm 5.4$ & $12.9 \pm 5.0$ & $12.8 \pm 4.8$ & 0.51 \\
\hline Physical inactivity, $n(\%)$ & $224(59.4)$ & $252(55.0)$ & $72(53.7)$ & 0.28 \\
\hline High alcohol consumption, $n(\%)^{\mathrm{d}}$ & $85(22.6)$ & $87(19.0)$ & $20(14.9)$ & 0.25 \\
\hline Current smoking, $n(\%)$ & $62(16.5)$ & $42(9.2)$ & $15(11.2)$ & 0.04 \\
\hline Parental diabetes with onset $<60$ years, $n(\%)$ & $39(10.3)$ & $33(7.2)$ & $6(4.5)$ & 0.16 \\
\hline $\mathrm{HbA}_{1 \mathrm{c}}, \%$ & $5.82 \pm 0.74$ & $5.77 \pm 0.80$ & $5.66 \pm 0.56$ & 0.10 \\
\hline $\mathrm{HbA}_{1 \mathrm{c}}, \mathrm{mmol} / \mathrm{mol}$ & $40.1 \pm 8.1$ & $39.6 \pm 8.7$ & $38.4 \pm 6.1$ & \\
\hline NGT at baseline, $n(\%)$ & $207(54.9)$ & $285(62.2)$ & $87(64.9)$ & 0.02 \\
\hline Prediabetes at baseline, $n(\%)$ & $99(26.3)$ & $109(23.8)$ & $32(23.9)$ & 0.61 \\
\hline Type 2 diabetes at baseline, $n(\%)$ & $71(18.8)$ & $64(14.0)$ & $15(11.2)$ & 0.03 \\
\hline Previously known & $46(12.2)$ & $36(7.9)$ & $10(7.5)$ & \\
\hline Newly detected & $25(6.6)$ & $28(6.2)$ & $5(3.7)$ & \\
\hline
\end{tabular}

Data are presented as means \pm SD for continuous traits or count (percentage) for categorical data

${ }^{a}$ Adjusted for age and sex

${ }^{\mathrm{b}}$ Hypertension is defined by: (1) blood pressure of 140/90 $\mathrm{mmHg}$; or (2) by antihypertensive medication under the condition that individuals report that they are hypertensive

${ }^{\mathrm{c}}$ SES was scored according to the Helmert Index [12]

${ }^{\mathrm{d}}$ High alcohol intake: $>40 \mathrm{~g} /$ day for men, $>20 \mathrm{~g} /$ day for women

Table 2 Association of the number of long-lived parents with prevalent prediabetes, prevalent type 2 diabetes and prevalent dysglycaemia ${ }^{\mathrm{a}}$ using logistic regression models: the KORA S4 study

\begin{tabular}{|c|c|c|c|c|c|c|c|}
\hline $\operatorname{Model}^{\mathrm{b}}$ & $\begin{array}{l}\text { Number of } \\
\text { long-lived } \\
\text { parents }\end{array}$ & $\begin{array}{l}n \text { (cases of } \\
\text { prediabetes) }\end{array}$ & $\begin{array}{l}\text { Prevalent } \\
\text { prediabetes }^{\mathrm{c}} \\
\text { OR }(95 \% \mathrm{CI})\end{array}$ & $\begin{array}{l}n \text { (cases of } \\
\text { diabetes) }\end{array}$ & $\begin{array}{l}\text { Prevalent type } 2 \\
\text { diabetes }^{\mathrm{c}} \\
\text { OR }(95 \% \mathrm{CI})\end{array}$ & $\begin{array}{l}n \text { (cases of } \\
\text { dysglycaemia) }\end{array}$ & $\begin{array}{l}\text { Prevalent } \\
\text { dysglycaemia }^{\mathrm{d}} \\
\text { OR }(95 \% \mathrm{CI})\end{array}$ \\
\hline \multirow[t]{3}{*}{ Model 1} & 2 & $134(32)$ & $0.71(0.44,1.15)$ & $134(15)$ & $0.46(0.25,0.85)$ & $134(47)$ & $0.61(0.40,0.92)$ \\
\hline & 1 & 458 (109) & $0.77(0.56,1.08)$ & $458(64)$ & $0.63(0.43,0.93)$ & $458(173)$ & $0.72(0.54,0.95)$ \\
\hline & 0 (reference) & 377 (99) & 1 & 377 (71) & 1 & $377(170)$ & 1 \\
\hline \multirow[t]{3}{*}{ Model 2} & 2 & $134(32)$ & $0.71(0.44,1.16)$ & $134(15)$ & $0.47(0.25,0.90)$ & $134(47)$ & $0.62(0.40,0.96)$ \\
\hline & 1 & $452(108)$ & $0.78(0.56,1.10)$ & $452(62)$ & $0.68(0.45,1.02)$ & $452(170)$ & $0.74(0.55,0.998)$ \\
\hline & 0 (reference) & $373(99)$ & 1 & $373(69)$ & 1 & $373(168)$ & 1 \\
\hline \multirow[t]{3}{*}{ Model 3} & 2 & $134(32)$ & $0.71(0.44,1.17)$ & $134(15)$ & $0.54(0.28,1.04)$ & $134(47)$ & $0.65(0.42,1.01)$ \\
\hline & 1 & $452(108)$ & $0.78(0.56,1.09)$ & $452(62)$ & $0.71(0.47,1.08)$ & $452(170)$ & $0.75(0.56,1.02)$ \\
\hline & 0 (reference) & 373 (99) & 1 & $373(69)$ & 1 & $373(168)$ & 1 \\
\hline
\end{tabular}

${ }^{\mathrm{a}}$ Dysglycaemia is defined as prediabetes or type 2 diabetes

${ }^{\mathrm{b}}$ Model 1, adjusted for age at baseline and sex; model 2, adjusted for age at baseline, sex, BMI, SES, smoking, alcohol consumption and physical activity; model 3, as for model 2 with additional adjustment for parental diabetes at onset $<60$ years

${ }^{\mathrm{c}}$ Results from multinomial logistic regressions, with reference group NGT

${ }^{\mathrm{d}}$ Results from binomial logistic regressions, with reference group NGT 
Table 3 Association of the number of long-lived parents with incident prediabetes, incident type 2 diabetes and incident dysglycaemia ${ }^{a}$ in participants with NGT at baseline using logistic regression models: the KORA S4/F4 cohort study

\begin{tabular}{|c|c|c|c|c|c|c|c|}
\hline Model $^{\mathrm{b}}$ & $\begin{array}{l}\text { Number of } \\
\text { long-lived } \\
\text { parents }\end{array}$ & $\begin{array}{l}n \text { (events of } \\
\text { prediabetes) }\end{array}$ & $\begin{array}{l}\text { Incident } \\
\text { prediabetes }^{c} \\
\text { OR }(95 \% \mathrm{CI})\end{array}$ & $\begin{array}{l}n \text { (events of } \\
\text { diabetes) }\end{array}$ & $\begin{array}{l}\text { Incident type } 2 \\
\text { diabetes }^{\mathrm{c}} \\
\text { OR }(95 \% \mathrm{CI})\end{array}$ & $\begin{array}{l}n \text { (events of } \\
\text { dysglycaemia) }\end{array}$ & $\begin{array}{l}\text { Incident } \\
\text { dysglycaemia }^{d} \\
\text { OR }(95 \% \text { CI })\end{array}$ \\
\hline \multirow[t]{3}{*}{ Model 1} & 2 & $59(9)$ & $0.42(0.19,0.94)$ & $59(3)$ & $0.65(0.16,2.62)$ & 59 (12) & $0.46(0.22,0.96)$ \\
\hline & 1 & $214(47)$ & $0.65(0.39,1.06)$ & $214(9)$ & $0.64(0.23,1.74)$ & $214(56)$ & $0.65(0.40,1.03)$ \\
\hline & 0 (reference) & $156(43)$ & 1 & $156(8)$ & 1 & $156(51)$ & 1 \\
\hline \multirow[t]{3}{*}{ Model 2} & 2 & $59(9)$ & $0.41(0.18,0.94)$ & $59(3)$ & $0.75(0.17,3.30)$ & $59(12)$ & $0.46(0.22,0.97)$ \\
\hline & 1 & $212(46)$ & $0.64(0.38,1.06)$ & $212(9)$ & $0.66(0.22,1.94)$ & $212(55)$ & $0.64(0.39,1.04)$ \\
\hline & 0 (reference) & $155(43)$ & 1 & $155(7)$ & 1 & $155(50)$ & 1 \\
\hline \multirow[t]{3}{*}{ Model 3} & 2 & $59(9)$ & $0.41(0.18,0.94)$ & $59(3)$ & $0.75(0.17,3.29)$ & $59(12)$ & $0.46(0.22,0.97)$ \\
\hline & 1 & $212(46)$ & $0.64(0.38,1.06)$ & $212(9)$ & $0.66(0.22,1.95)$ & $212(55)$ & $0.64(0.39,1.04)$ \\
\hline & 0 (reference) & $155(43)$ & 1 & $155(7)$ & 1 & $155(50)$ & 1 \\
\hline
\end{tabular}

\footnotetext{
${ }^{a}$ Dysglycaemia is defined as prediabetes or type 2 diabetes

${ }^{\mathrm{b}}$ Model 1, adjusted for age at baseline and sex; model 2, adjusted for age at baseline, sex, BMI, SES, smoking, alcohol consumption and physical activity; model 3, as for model 2 with additional adjustment for parental diabetes at onset $<60$ years

${ }^{\mathrm{c}}$ Results from multinomial logistic regressions, with reference group NGT

${ }^{\mathrm{d}}$ Results from binomial logistic regressions, with reference group NGT
}

of dysglycaemia was borderline significant in participants with only one long-lived parent (OR $0.65,95 \%$ CI 0.40 , 1.03). These associations were virtually unchanged after further adjustment for BMI, SES, lifestyle factors and early onset of diabetes in the parents. The mean parental age at death per 10-year increase was not significantly associated with incident dysglycaemia (OR $0.84,95 \%$ CI $0.66,1.06$ ).

\section{Discussion}

This study showed that parental longevity, defined by a lifespan of at least 80 years, was associated with a lower prevalence and incidence of dysglycaemia in an older German population. Dose-effect relationships were observed between the number of long-lived parents and the prevalence of type 2 diabetes, and between the number of long-lived parents and the risk of dysglycaemia.

So far, parental longevity has been shown to reduce the risk of diabetes in their offspring in only one prospective study with a selected sample of participants from the Diabetes Prevention Program study with a high diabetes risk [9]. To our knowledge, our study is the first population-based study to find an association between parental longevity and incident dysglycaemia.

In contrast to other cross-sectional studies that have included the offspring of centenarians or of nonagenarian siblings [3-7], we applied less rigid criteria to define longevity in the present study. Even having only one parent who had died at age 80 or later was associated with a reduced prevalence of diabetes. The findings of an association between moderate parental longevity and the prevalence or incidence of dysglycaemia are unlikely to be accidental for several reasons. First, effects were fairly strong, as can be seen, for example, by an OR of 0.46 describing the reduced prevalence of diabetes in individuals with two compared with no long-lived parents. Second, a lower prevalence of dysglycaemia was independently observed both in those with long-lived mothers and in those with long-lived fathers. Third, as already mentioned, the effects were stronger in participants with two rather than one long-lived parent.

In our analyses, we first fitted models with adjustment for age and sex. Next, we fitted models with adjustment for potential mediators between parental longevity and diabetes in the offspring. In a first step, we considered lifestyle factors, BMI and SES; in a second step, we additionally adjusted our models for onset of diabetes in the parents before the age of 60 years as a marker of genetic factors.

Surprisingly, adjustment for lifestyle factors did not attenuate the associations between parental longevity and prevalence of diabetes. One explanation might be that the lifestyle of many descendants has become very different from that of their parents owing to a rapid social change over the post-war decades, so that the lifestyle of the KORA participants is hardly associated with the lifestyle of their parents. Furthermore, model variables might not have been adequate enough to describe the lifestyle of the participants (for example, no dietary variables and no assessment of physical activity at work).

Adjustment for parental diabetes with an onset before the age of 60 also did not strongly attenuate the associations between parental longevity and the prevalence or incidence of diabetes in their offspring. Adjusting for early parental diabetes instead of adjusting for any parental diabetes might 
have the disadvantage of neglecting most cases of parental diabetes that occur after the age of 60 . However, adjustment for parental diabetes with onset at any age might lead to an even greater bias because the diagnosis of diabetes is more likely in long-lived than short-lived individuals, and thus parental diabetes with an onset at any age mainly reflects length of life rather than genetic factors.

To explain the association between family longevity and prevalence of type 2 diabetes, the frequency of 15 risk alleles known to be related to type 2 diabetes was compared between the offspring of nonagenarian siblings and the offspring's partners in the Leiden Longevity Study [13]. Although the offspring had a better glucose metabolism than their partners, there were no differences in the frequencies of any risk allele, and the mean numbers of risk alleles were virtually identical in both groups. To explain this finding, the authors assume that the risk alleles included in the analyses were mainly related to beta cell function, whereas the better glucose metabolism in the offspring was due to better insulin sensitivity. Alternatively, further results from the Leiden Longevity Study suggest that alleles protecting from disease rather than risk alleles may determine longevity [14]: long-lived individuals and younger controls did not differ in the number of risk alleles associated with cardiovascular disease, cancer and diabetes, and protective alleles may have a stronger effect on longevity than cumulated risk alleles.

Genetic factors may also contribute to better profiles of diabetes risk factors in the offspring of long-lived families: In a rather small study group, the offspring of Ashkenazi Jewish centenarians were shown to have inherited more favourable lipid profiles [15], and a reduced prevalence of hypertension was observed in the offspring of nonagenarian siblings and the offspring of centenarians [4-6].

For a deeper understanding of the genetic and environmental factors leading to a lower prevalence and incidence of dysglycaemia in persons with long-lived parents, further research is necessary. Risk alleles related to insulin secretion and to insulin sensitivity, as well as protective alleles, have to be taken into account. Moreover, a sound assessment of lifestyle factors including diet and physical activity at work is necessary, and one might also consider gene-environment interactions.

This study had some limitations. As mentioned above, lifestyle variables might not have been assessed adequately enough to confirm whether components of lifestyle are mediators of the association between parental longevity and prevalence of diabetes. Longitudinal associations between parental longevity and dysglycaemia were assessed, but there was little power for the analysis of associations between parental longevity and incidence of type 2 diabetes. A strength of our study is the well-defined population sample. Contrary to other studies, we excluded individuals whose parents had lost their lives due to traumatic causes of death. Moreover, glucose tolerance tests were carried out to assess prediabetes and type 2 diabetes at baseline and at follow-up. In addition, we did not focus on extreme longevity, so that our results apply to a larger number of persons.

In conclusion, the prevalence of diabetes is associated not only with extreme family longevity as previously shown, but also with moderate parental longevity as shown in this study. Furthermore, this is the first study to find an association between parental longevity and incidence of dysglycaemia.

Acknowledgements We thank the field staff in Augsburg who were involved in conducting the studies.

Funding The Diabetes Cohort Study was funded by a German Research Foundation project grant to WR (DFG; RA 459/2-1). The German Diabetes Center is funded by the German Federal Ministry of Health and the Ministry of School, Science and Research of the State of North-Rhine-Westfalia. The KORA research platform and the KORA Augsburg studies are financed by the Helmholtz Zentrum München, German Research Center for Environmental Health, which is funded by the German Federal Ministry of Education, Science, Research and Technology and by the State of Bavaria. The KORAAge project was financed by the German Federal Ministry of Education and Research (BMBF FKZ 01ET0713) as part of the programme Health in Old Age.

Duality of interest The authors declare that there is no duality of interest associated with this manuscript.

Contribution statement WR and BK planned the study. WR, AP, $\mathrm{BT}$ and $\mathrm{CM}$ contributed to the acquisition of data. $\mathrm{BK}$ analysed the data and wrote the manuscript. WR, AP, BT and CM contributed to interpretation of the results and revision of the manuscript. All authors provided final approval of the manuscript.

\section{References}

1. The Emerging Risk Factors Collaboration (2011) Diabetes mellitus, fasting glucose, and risk of cause-specific death. N Engl J Med 364:829-841

2. Galioto A, Dominguez LJ, Pineo A et al (2008) Mini review: cardiovascular risk factors in centenarians. Exp Gerontol 43:106-113

3. Westendorp RGJ, van Heemst D, Rozing MP et al (2009) Nonagenarian siblings and their offspring display lower risk of mortality and morbidity than sporadic nonagenarians: the Leiden Longevity Study. J Am Geriatr Soc 57:1634-1637

4. Rozing MP, Westendorp RGJ, de Craen AJM et al (2010) Favorable glucose tolerance and lower prevalence of metabolic syndrome in offspring without diabetes mellitus of nonagenarian siblings: the Leiden Longevity Study. J Am Geriatr Soc 58:564569

5. Atzmon G, Schechter C, Greiner W, Davidson D, Rennert G, Barzilai N (2004) Clinical phenotype of families with longevity. J Am Geriatr Soc 52:274-277

6. Terry DF, Wilcox M, McCormick MA, Lawler E, Perls TT (2003) Cardiovascular advantages among the offspring of centenarians. J Gerontol A Biol Sci Med Sci 58:425-431 
7. Terry DF, Wilcox MA, McCormick MA, Perls TT (2004) Cardiovascular disease delay in centenarian offspring. J Gerontol A Biol Sci Med Sci 59:385-389

8. Frederiksen H, McGue M, Jeune B et al (2002) Do children of long-lived parents age more successfully? Epidemiology 13:334-339

9. Florez H, Ma Y, Crandall JP et al (2011) Parental longevity and diabetes risk in the Diabetes Prevention Program. J Gerontol A Biol Sci Med Sci 66:1211-1217

10. Rathmann W, Haastert B, Icks A et al (2003) High prevalence of undiagnosed diabetes mellitus in Southern Germany: target populations for efficient screening. The KORA Survey 2000. Diabetologia 46:182-189

11. World Health Organization (2006) Definition and diagnosis of diabetes mellitus and intermediate hyperglycemia. Report of a WHO/IDF consultation. WHO, Geneva
12. Kowall B, Rathmann W, Strassburger K, Meisinger C, Holle R, Mielck A (2011) Socioeconomic status is not associated with type 2 diabetes incidence in an elderly population in Germany: KORA S4/F4 Cohort Study. J Epidemiol Community Health 65:606-612

13. Mooijaart SP, van Heemst D, Noordam R et al (2011) Polymorphisms associated with type 2 diabetes in familial longevity: the Leiden Longevity Study. Aging 3:55-62

14. Beekman M, Nederstigt C, Suchiman HED et al (2010) Genomewide association study (GWAS)-identified disease risk alleles do not compromise human longevity. Proc Natl Acad Sci USA 107:18046-18049

15. Barzilai N, Gabriely I, Gabriely M, Iankowitz N, Sorkin JD (2001) Offspring of centenarians have a favorable lipid profile. J Am Geriatr Soc 49:76-79 